\title{
Der Nutzen der Dekompression wird durch eine zusätzliche Fusionsoperation nicht erhöht
}

Fragestellung: Ist bei Patienten mit lumbaler Spinalkanalstenose und Claudicatio spinalis die reine Dekompressionsoperation (Laminektomie) ebenso wirksam wie eine Laminektomie mit einer Fusionsoperation?

Hintergrund: Bei vielen älteren Menschen kommt es im Rahmen von degenerativen Veränderungen der Lendenwirbelsäule zu einer lumbalen Spinalkanalstenose mit lokalen und radikulären Schmerzen sowie zu einer Claudicatio spinalis. Wenn diese Symptome ausgeprägt und anhaltend sind und durch eine konservative Therapie nicht gebessert werden können, verbleibt nur die Option eines operativen Eingriffs. Hier stehen zwei Möglichkeiten zur Verfügung: zum einen die reine Laminektomie, um den komprimierten lumbalen und sakralen Wurzeln Platz zu verschaffen, und zum anderen die Laminektomie in Kombination mit einer Fusionsoperation mit Schrauben und Titanstäben. Eine gute randomisierte Studie, die diese beiden Operationstechniken vergleicht, gab es bisher nicht.

Patienten und Methodik: Die schwedischen Neurochirurgen randomisierten in diese Studie 247 Patienten im Alter zwischen 50 und 80 Jahren, die über ein oder zwei Segmente eine lumbale Spinalkanalstenose und lang anhaltende Beschwerden aufwiesen. Die Patienten erhielten entweder eine reine De-

Försth P, Ólafsson G, Carlsson T et al. A randomized, controlled trial of fusion surgery for lumbar spinal stenosis. N Engl J Med 2016; 374: $1413-23$ se vorlag. Als Outcome-Parameter dienten der 6-Minuten-Gehtest und eine gesundheitsökonomische Evaluation. Der primäre Endpunkt war der Wert auf dem Oswestry Disability Index (Skala von 0 bis 100), der die durch die chronischen Rückenschmerzen verursachte Behinderung misst. Der primäre Endpunkt wurde zwei Jahre nach der Operation erhoben.

Ergebnisse: Die Patienten waren im Mittel 66 Jahre alt und hatten einen Wert auf dem Oswestry Disability Index von im Mittel 41. Der visuelle Analogscore für Rückenschmerzen auf der Skala von 0 bis 100 lag bei 60 und für Schmerzen ausstrahlend in das Bein bei 62. Die zurückgelegte Strecke im 6-MinutenGehtest betrug im Schnitt 320 Meter. Von den ursprünglich 247 randomisierten Patienten wurden 113 laminektomiert und einer Fusionsoperation unterzogen und 120 nur laminektomiert. Für die endgültige Analyse nach zwei Jahren standen 111 Patienten in der ersten Gruppe und 117 in der zweiten Gruppe zur Verfügung. Für keinen der primären und sekundären Endpunkte ergab sich nach zwei Jahren ein Unterschied zwischen den beiden Operationsverfahren. Dies galt auch für Patienten, die über einen Zeitraum von fünf Jahren nachbeobachtet wurden. In der Fusionsgruppe dauerte allerdings die Operation signifikant länger und es kam auch zu einem höheren Blutverlust. Die Operation als solche war bei den meisten Patienten erfolgreich, so gaben 60 - 80\% der Patienten eine signifikante Besserung der Rücken- und der radikulären Schmerzen an.

Schlussfolgerungen: Bei Patienten mit lumbaler Spinalkanalstenose und Claudicatio spinalis ist die reine Dekompressionsoperation mit Laminektomie genauso wirksam wie eine Laminektomie mit einer Fusionsoperation.

\section{Diese Erkenntnisse müssen in die Beratung des Patienten einfließen}

\begin{abstract}
Die Ergebnisse dieser großen schwedischen Studie haben erhebliche Implikationen für das Gesundheitssystem. In Deutschland werden bei Spinalkanalstenosen durch Neurochirurgen überwiegend minimal-invasiv und mikrochirurgisch Dekompressionen vorgenommen, währenddessen durch Wirbelsäulenchirurgen und Orthopäden viel häufiger neben der Dekompression eine Fusionsoperation erfolgt, die länger dauert, mit mehr Komplikationen behaftet ist und deutlich teurer ist. Sie wird allerdings im DRG-System auch deutlich besser vergütet. Die schwedische Studie zeigt, dass ganz offenbar bei den meisten Patienten die reine Dekompressionsoperation ausreicht und eine hohe Erfolgsquote hat. Die Ergebnisse dieser Studie müssen in die Beratung von Patienten, die zur Einholung einer zweiten Meinung den Neurologen aufsuchen, einfließen.
\end{abstract}

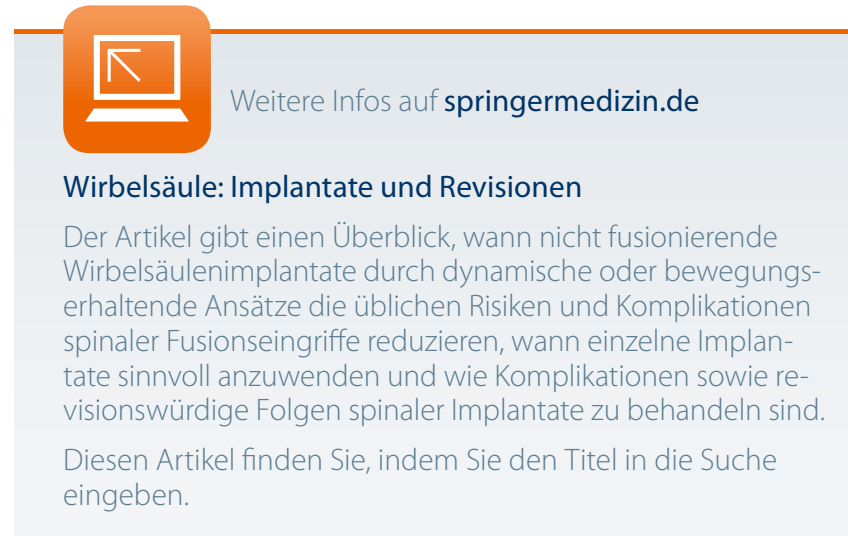

